\title{
A NEW ALGEBRAIC APPROACH TO MICROLOCALIZATION OF FILTERED RINGS
}

\author{
MARIA J. ASENSIO, MICHEL VAN DEN BERGH AND FREDDY VAN OYSTAEYEN
}

\begin{abstract}
Using the construction of the Rees ring associated to a filtered ring we provide a description of the microlocalization of the filtered ring by using only purely algebraic techniques.

The method yields an easy approach towards the study of exactness properties of the microlocalization functor. Every microlocalization at a regular multiplicative Ore set in the associated graded ring can be obtained as the completion of a localization at an Ore set of the filtered ring.
\end{abstract}

\section{INTRODUCTION}

Microlocal differential operators appear in the theory of $D$-modules where they are defined and treated by analytic methods. In search of an algebraic theory of microlocalization, T. Springer [7] introduced algebraic microlocalization of a filtered ring $A$ at a multiplicatively closed subset $S$ in $A$ in the case where the associated graded ring $G(A)$ is a commutative domain. The technique could be extended to microlocalization at a multiplicative subset $S$ of $A$ such that $\sigma(S)$ in $G(A)$ is a left Ore set without further restrictions on the filtered ring $A$ (cf. [8]). The algebraic theory expounded in loc. cit. is not as "algebraic" as most algebraists would like because the methods and results all depend on rather technical calculations with pseudo-norms and completions for these.

In this paper we present a purely algebraic approach by passing through the Rees ring $\widetilde{R}=\bigoplus_{n \in \mathbf{Z}} F_{n} R$, associated to the filtration $\left\{F_{n} R, n \in \mathbf{Z}\right\}$ on the ring $R$, and establishing an equivalence of categories between the category $R$ filt of filtered left $R$-modules and $\mathscr{F}_{X}$, the category of $X$-torsion free graded $\widetilde{R}$-modules where $X$ stands for the element of $\widetilde{R}$ representing 1 as an element of $F_{1} R=\widetilde{R}_{1}$. The relations $\widetilde{R} /(X) \cong G(R), \widetilde{R} /(X-1) \cong R$, and $\widetilde{R}_{X} \cong$ $R\left[X, X^{-1}\right]$ allow the interesting interplay on which the results in this paper depend. It is clear that filtered properties of objects in $R$-filt will translate to graded properties in $\mathscr{F}_{X}$ and vice versa; this in its own right provides interesting applications, short elegant proofs of earlier results as well as new perspectives.

Received by the editors September 17, 1987.

1980 Mathematics Subject Classification (1985 Revision). Primary 16A80; Secondary 13J10, $16 \mathrm{~A} 08$.

The second author was supported by an NFWO grant. 
The body of the paper deals with the formulation of microlocalization in the category $\widetilde{R}$-gr stressing its homological properties.

In the theory of $D$-modules the sheaves of microlocal differential operators are of main interest, therefore it makes sense to consider microlocalizations associated to open sets (that are not necessarily corresponding to multiplicity closed sets) and we point out briefly how our theory extends to that situation (for algebraic microlocalization at $S$ this was not possible in the earlier formalism).

One of the interesting results of this paper is Theorem 3.19(2) where we establish flatness of microlocalization at a multiplicative set $S$; it follows from this that for regular saturated multiplicative sets $S_{\text {sat }}$ in a filtered ring $R$, subjected only to the condition that $\widetilde{R}$ is Noetherian, the microlocalization at $S_{\text {sat }}$ equals the completion of $S_{\text {sat }}^{-1} R$ (hence in this case $S_{\text {sat }}$ is automatically a real Ore set).

For general theory of filtered rings we refer to [2 and 6], the second is also our basic reference for results concerning graded rings. We repeat definitions and some fundamental facts in $\S 1$, develop the constructions and their general properties that we need in $\S 2$, and focus on microlocalization in $\S 3$.

\section{Preliminaries}

1.1. All rings are associated with identity. A module is always a left module unless otherwise mentioned. A filtration on a ring $R$ is given by an ascending chain of additive subgroups $\left\{F_{n} R, n \in \mathbf{Z}\right\}$ such that $F_{n} R F_{m} R \subset F_{n+m} R, 1 \in$ $F_{0} R$, for all $n, m \in \mathbf{Z}$. An $R$-module $M$ is a filtered module if there is an ascending chain of additive subgroups $\left\{F_{n} M, n \in \mathbf{Z}\right\}$ satisfying $F_{n} R F_{m} M \subset$ $F_{n+m} M$ for every $n, m \in \mathbf{Z}$. Throughout this paper all filtrations considered will be exhaustive, i.e. $\bigcup_{n} F_{n} R=R$ and $\bigcup_{n} F_{n} M=M$.

A filtration $F M$ (short for $\left\{F_{n} M, n \in \mathbf{Z}\right\}$ ) is discrete if there is an $n_{0} \in \mathbf{Z}$ such that $F_{j} M=0$ for all $j<n_{0}$. A filtration $F R$ is separated if $\bigcap_{n} F_{n} M=0$. The trivial filtration on $M$ is given by $F_{t} M=0$ for $t<0$ and $F_{s} M=M$ for $s \geq 0$. An $R$-linear map between $R$-modules $M$ and $N, f$ say, is said to be a filtered morphism of degree $p \in \mathbf{Z}$ if $f\left(F_{i} M\right) \subset F_{i+p}(N)$ for all $i \in \mathbf{Z}$. Homomorphisms of finite degree form a subgroup $\operatorname{HOM}_{R}(M, N)$ of $\operatorname{Hom}_{R}(M, N)$ that may be filtered by taking for $F_{p} \operatorname{HOM}_{R}(M, N)$ the morphisms of degree $p$. We write $\operatorname{Hom}_{F R}(M, N)=F_{0} \operatorname{HOM}_{R}(M, N)$ for the morphisms in the category $R$-filt of filtered left $R$-modules. Arbitrary direct sums, direct products, direct limits and projective limits exist in $R$-filt; the category is pre-abelian but not abelian. An $M \in R$-filt is said to be complete if $F M$ is separated and all Cauchy sequences (for the topology of $F M$ ) converge in $M$. The completion of $M$ with respect to $F M$ is given by $\lim _{n} M / F_{n} M$ (for more detail cf. $\S I$, p. 283 of [6]).

1.2. A ring $A$ is said to be $\mathbf{Z}$-graded if $A=\bigoplus_{n \in \mathbf{Z}} A_{n}$, where the $A_{n}$ are additive subgroups satisfying $A_{n} A_{m} \subset A_{n+m}$ for all $n, m \in \mathbf{Z}$. A graded $A$ module $M$ is given by $M=\bigoplus_{n \in \mathbf{Z}} M_{n}$ with $A_{n} M_{m} \subset M_{n+m}$ and it is clear how 
to define graded morphisms, etc. (cf. [6]). The graded morphisms of degree zero from $M$ to $N$, where $M$ and $N$ are graded $R$-modules, are the morphisms in the category $A$-gr. This category is a Grothendieck category.

1.3. If $R$ is a filtered ring and $M \in R$-filt then we define the abelian groups

$$
G(R)=\bigoplus_{n \in \mathbf{Z}} F_{n} R / F_{n-1} R, \quad G(M)=\bigoplus_{n \in \mathbf{Z}} F_{n} M / F_{n-1} M .
$$

If $m \in F_{p} M$ then $m_{(p)}$ denotes the image of $m$ in $G(M)_{p}=F_{p} M / F_{p-1} M$. For $a \in F_{i} R$ and $m \in F_{j} M$ we define $a_{(i)} m_{(j)}=(a m)_{(i+j)}$ and extend it to a Z-bilinear $\mu: G(R) \times G(M) \rightarrow G(M)$. For $M=R, \mu$ makes $G(R)$ into a graded ring and in general $G(M)$ is a graded $G(R)$-module. It is easily checked that $G$ defines a functor $R$-filt $\rightarrow G(R)$-gr which commutes with direct sums, products, direct and projective limits. Clearly, if $F M$ is separated then $M=0$ if and only if $G(M)=0$.

A filtered morphism $f: M \rightarrow N$ is said to be a strict morphism if $f\left(F_{p} M\right)=$ $\operatorname{Im} f \cap F_{p} N$ for all $p \in \mathbf{Z}$. A sequence in $R$-filt:

$$
L \underset{f}{\rightarrow} M \underset{g}{\rightarrow} N
$$

is strict exact if it is an exact sequence viewed as a sequence in $R$-mod, such that $f$ and $g$ are strict morphisms in $R$-filt. A filtered morphism $f: M \rightarrow N$ is an isomorphism in $R$-filt if and only if $f$ is bijective and strict. Obviously, a surjection $f: M \rightarrow N$ is a strict morphism if and only if $F N$ is the quotient filtration defined by $f$, i.e. $F_{p} N=f\left(F_{p} M\right)$. If $(*)$ is a strict exact sequence then $G(*)$ is exact (cf. Theorem III.3, p. 288 in [6]). Hence by the foregoing remarks we have $G(M / N)=G(M) / G(N)$ if $M / N$ has the quotient filtration of $F M$ and $N$ has the induced filtration $F_{p} N=F_{p} M \cap N$ for all $p \in \mathbf{Z}$. Also, if $F M$ is separated then an $f \in \operatorname{Hom}_{F R}(M, N)$ is surjective and strict if and only if $G(f)$ is injective (note: the inclusion $M \subset N$ is strict if and only if $M$ has the induced filtration).

1.4. We say that $M \in R$-filt is filt-free if it is a free $R$-module having a basis $\left\{x_{i}, i \in \mathscr{T}\right\}$ such that $F_{p} M=\bigoplus_{i \in \mathcal{F}} F_{p-n_{i}} R \cdot x_{i}$ for some family of integers $\left\{n_{i}, i \in \mathscr{T}\right\}$. Note that 'filt-free' is stronger than being filtered and free as an $R$-module!

An $M \in R$-filt is said to be filt-finitely generated if for all $p \in \mathbf{Z}, F_{p} M=$ $\sum_{i=1}^{n} F_{p-n_{i}} R \cdot x_{i}$ for a finite family of $x_{i} \in M, n_{i} \in \mathbf{Z}$, or equivalently if there is a filt-free $L$ that is finitely generated together with a strict surjection $\pi: L \rightarrow M$. For a filt-finitely generated $M$ we have that $G(M)$ is a finitely generated graded $G(R)$-module but the converse is true only under some additional hypotheses (cf. Proposition IV.3 on p. 294 of [6] and Proposition 1.10 of [1]). If $M$ is filt-finitely generated then we say that $F M$ is good.

1.5. In the study of completions one uses a notion of equivalence for filtrations $F M$ and $F^{\prime} M$ on an $R$-module $M$ that is inspired by topological 
considerations only, i.e. $F M$ and $F^{\prime} M$ are said to be topologically equivalent if they determine the same topology, i.e. for each $s, t \in \mathbf{Z}$ there exist $s^{\prime}, t^{\prime} \in \mathbf{Z}$ such that $F_{s^{\prime}} M \subset F_{s}^{\prime} M, F_{t^{\prime}}^{\prime} M \subset F_{t} M$. For the study of finiteness properties, e.g. good filtrations, we introduce a stronger notion of equivalence that does reduce to the former only when the suitable finiteness conditions hold. The filtrations $F M$ and $F^{\prime} M$ on the $R$-module $M$ are said to be equivalent if there exists a positive integer $w$ such that $F_{m-u} M \subset F_{n}^{\prime} M \subset F_{n+w} M$ for all $n \in \mathbf{Z}$.

1.6. Proposition. If the $R$-module has a good filtration then all good filtrations on $M$ are equivalent.

Proof. Well known, cf. [2, 8].

1.7. In the next result we recall part of Theorem 2.11 of [1]. It shows that several conditions on filtrations appearing in the literature will actually come down to the same thing. Recall that $F M$ is said to be faithful if $G(M)=$ 0 implies $M=0 ; F R$ is a faithful filtration if $F M$ is faithful for every good filtration $F M$ on a finitely generated $R$-module $M$. We say that $F R$ is Zariskian if $G(R)$ is Noetherian and if for any pair $N \subset M$ of filt-finitely generated $R$-modules (i.e. with good filtrations) we have

$$
F_{n} N=\bigcap_{m \in \mathbf{Z}}\left(F_{n} N+F_{m} M\right) \quad \text { for all } n \in \mathbf{Z} \text {. }
$$

1.8. Theorem [1]. Let $R$ be a filtered ring such that $G(R)$ is left Noetherian. The following statements are equivalent:

(1) FR is faithful and good filtrations induce good filtrations.

(2) Good filtrations are separated and good filtrations induce good filtrations on finitely generated $R$-submodules.

(3) $F R$ is Zariskian.

(4) $F R$ is $\Sigma$-Noetherian in the sense of [8, Definition 6.10].

1.9. Example. If $F R$ is complete, and $G(R)$ is left Noetherian then the properties mentioned in the theorem do hold.

1.10. Remark. In [1] the list of equivalent conditions is somewhat longer but the omited conditions involved the comparison condition, and in [1] the proofs of equivalences involving the comparison condition have gaps. We will establish the result we actually need in Proposition 2.6 in terms of a finiteness condition on the Rees ring $\widetilde{R}$ associated to $F R$.

\section{Filtered modules as GRAded modules over the ReEs RiNG}

Let $R$ be a filtered ring (exhaustive, as usual), then $\widetilde{R}=\bigoplus_{n \in \mathrm{Z}} F_{n} R$ is obviously a $Z$-graded ring. We may also view $\widetilde{R}$ as a subring of $R\left[X, X^{-1}\right], X$ a variable, $\widetilde{R} \cong \sum_{n \in \mathbf{Z}} F_{n} R X^{n}$ with $X \in \widetilde{R}_{1}$. The canonical element of degree one in $\widetilde{R}$ is the $1 \in F_{1} R$ in $\widetilde{R}$, i.e. we write it as $X$ to conform with the second presentation of $\widetilde{R}$. The ideal generated in $\widetilde{R}$ by the central element $X$, i.e. 
$\sum_{n \in \mathrm{Z}} F_{n} R X^{n+1}$, will be denoted by $I$ throughout this paper. We let $\widetilde{F} \widetilde{R}$ be the $I$-adic filtration on $\widetilde{R}, \widetilde{F}_{n} \widetilde{R}=I^{-n}$ for $n<0$ and $\widetilde{F}_{m} \widetilde{R}=\widetilde{R}$ for $m \geq 0$. If $M$ is a filtered $R$-module then $\widetilde{M}=\bigoplus_{n \in \mathbf{Z}} F_{n} M$ is in the obvious way a graded $\widetilde{R}$-module (and it may also be viewed as $\widetilde{M}=\sum_{n \in \mathbf{Z}} F_{n} M X^{n}$ ). Obviously each $\widetilde{M}$ is $X$-torsionfree because the maps $F_{n} M \rightarrow F_{n+1} M$ are injective.

Conversely, to an $X$-torsionfree graded $\widetilde{R}$-module $\widetilde{M}$ there corresponds a filtered $R$-module $M$ that is obtained as $\lim _{n} \widetilde{M}_{n}$ where the maps in the inductive system are given by 'multiplication by $X$ ' and these are injective. The canonical images of $\widetilde{M}_{n}$ in $M$ then define an exhaustive filtration on $M$ (the $R$-module structure is obvious). All these observations may be combined in the following lemma; all claims are easily verified or just immediate consequences of the definitions.

2.1. Lemma. Let $R$ be a filtered ring, $\widetilde{M} \in \mathscr{F}_{X}$, the latter being the full subcategory in $\widetilde{R}$-gr of the $X$-torsionfree graded $\widetilde{R}$-modules.

(1) $\widetilde{R} / I \cong G(R), \widetilde{M} / I \widetilde{M} \cong G(M)$.

(2) $\widetilde{R} / \widetilde{R}(X-1) \cong R, \widetilde{M} /(X-1) \widetilde{M} \cong M$; as observed earlier we may use multiplication by $X$ as an identification map in constructing $M$ from $\widetilde{M}$.

(3) The localization of $\widetilde{R}$ at the central multiplicatively closed set $\{1, X$, $\left.X^{2}, \ldots\right\}$, denoted by $\widetilde{R}_{X}$, equals $R\left[X, X^{-1}\right]$. Also $\widetilde{M}_{X} \cong M\left[X, X^{-1}\right]$ (uses the fact that $F M$ is exhaustive).

(4) The functor $\sim: R$-filt $\rightarrow \widetilde{R}$-gr defines an equivalence of categories between $R$-filt and $\mathscr{F}_{X}$.

Making use of the correspondence between $R, \widetilde{R}$ and $G(R)$, in particular Lemma $2.1(4)$, it is straightforward to verify the properties mentioned in the following lemmas.

2.2. Lemma. With notations as in the foregoing lemma:

(1) For an $M \in R$-filt, $F M$ is a separated filtration if and only if the I-adic filtration on $\widetilde{M}$ is separated, i.e. when $\bigcap_{n} X^{n} \widetilde{M}=0$.

(2) A filtered $R$-module $M$ is filt-finitely generated (or equivalently $F M$ is good) if and only if $\widetilde{M}$ is finitely generated as a graded $\widetilde{R}$-module.

(3) A filtered R-module $M$ is filt-free (e.g. as defined in [6]) if and only if $\widetilde{M}$ is gr-free in $\widetilde{R}$-gr.

(4) A filtered morphism $f: M \rightarrow N$ in $R$-filt is a strict morphism if and only if $\tilde{f}: \widetilde{M} \rightarrow \widetilde{N}$ is a graded morphism (of degree zero) such that $\operatorname{Coker}(\tilde{f}) \in \mathscr{F}_{X}$. So if $f$ is strict then $\operatorname{Ker}(\tilde{f})$ and $\operatorname{Coker}(\tilde{f})$ are both in $\mathscr{F}_{X}$. A strict exact sequence in $R$-filt transforms to a graded exact sequence in $\mathscr{F}_{X}$.

2.3. Lemma. (1) If $P \in R$-filt is filt-projective (the definition is the obvious one, see also $\S \mathrm{VI}, p .306,[6])$ then $\widetilde{P}$ is gr-projective and conversely.

(2) If $E \in R$-filt is an injective object then $\widetilde{E}$ is an injective object of $\mathscr{F}_{X}$. 
2.4. Note. 1. In Lemma 2.3(1) one should bear in mind Corollary I.2.2, p. 7 of [6], yielding that the notion of gr-projectivity is the same as projectivity for any graded module.

2. In Lemma 2.3(2), $\widetilde{E}$ need not be injective in $\widetilde{R}$-mod and in fact not even gr-injective in $\widetilde{R}$-gr.

3. Lemma 2.1 provides methods for transporting properties from $M$ to $G(M)$ and vice versa, usually through $\widetilde{M}$. Let us just mention here that CohenMacaulay properties may be studied this way because we go from $\widetilde{R}$ to $G(R)$ by reducing modulo a central element. Such lifting-and descent-results depend on properties of the functors: $-\otimes_{\widetilde{R}} \widetilde{R} / I$ and $-\otimes_{\widetilde{R}} \widetilde{R} /(X-1) \widetilde{R}$. To this end we include

2.5. Lemma. The functor $\widetilde{G}=-\otimes_{\widetilde{R}} \widetilde{R} / I$ is exact on $\mathscr{F}_{X}$; the functor $-\otimes_{\widetilde{R}} \widetilde{R} /(X-1) \widetilde{R}$ is exact on $\widetilde{R}$-gr.

Proof. Easy.

Let us investigate in some detail when $\widetilde{R}$ is left Noetherian. Since $\widetilde{R}$ is $\mathbf{Z}$ graded we know that $\widetilde{R}$ is left Noetherian if and only if it is left gr-Noetherian, in view of Theorem II.3.5 on p. 88 in [6]. In view of Lemma 2.2(2) we see that $\widetilde{R}$ is gr-Noetherian if and only if every filtered submodule $N$ of a filt-free $R$ module $E$ of finite rank is filt-finitely generated. Note that $F N$ is not induced by $F E$ unless $\widetilde{E} / \widetilde{N}$ is $X$-torsionfree (cf. Lemma 2.2(4)). Because of this fact the equivalence established in Proposition 1.1.7, p. 57 in [10] is wrong; of course the condition that $\widetilde{R}$ is left Noetherian will entail that good filtrations induce good filtrations on submodules but the converse will hold only in the presence of the condition $G(R)$ Noetherian. In view of Lemma 2.1(4) we do obtain that $R$ is left Noetherian if and only if $\widetilde{R}$ is $I$-Noetherian, that is $\widetilde{R}$ then satisfies the ascending chain condition with respect to $X$-closed left ideals, but for $\widetilde{R}$ to be left Noetherian we really do need extra assumptions.

2.6. Proposition. Let $R$ be a filtered ring. Then the following are equivalent:

1. (a) Good filtrations induce good filtrations on R-submodules.

(b) $G(R)$ is Noetherian.

2. $\widetilde{R}$ is Noetherian.

Proof. First note that condition 1(a) is equivalent with the following:

If $\widetilde{M} \subset \widetilde{N}$ are in $\mathscr{F}_{X}$ with $\widetilde{N}$ finitely generated and such that $\widetilde{N} / \widetilde{M}$ is in $\mathscr{F}_{X}$, then $\widetilde{M}$ is finitely generated.

$2 \Rightarrow 1$. This is clear.

$1 \Rightarrow 2$. Let $\widetilde{M} \subset \widetilde{N}$ be in $\mathscr{F}_{X}$ with $\widetilde{N}$ finitely generated. Then we claim that $\widetilde{M}$ is finitely generated. This proves that $\widetilde{R}$ is Noetherian since we may take $\widetilde{N}=\widetilde{R}$.

If $\widetilde{M}^{\prime}$ is the graded module such that $\widetilde{M} \subset \widetilde{M}^{\prime} \subset \widetilde{N}$ and $\widetilde{N} / \widetilde{M}^{\prime}$ is in $\mathscr{F}_{X}$ then $\widetilde{M}^{\prime}$ is finitely generated by $1(\mathrm{a})$. Hence we may assume that $\widetilde{N} / \widetilde{M}$ is 
$X$-torsion. It follows that there is some $u$ such that $X^{u} \widetilde{N} \subset \widetilde{M}$. Hence $\widetilde{M}$ is an extension of $X^{u} \widetilde{N}$ and modules $U_{t}=X^{t} \widetilde{N} \cap \widetilde{M} / X^{t+1} \widetilde{N} \cap \widetilde{M}$ for $t=$ $0, \ldots, u-1$. Each $U_{t}$ embeds in $X^{t} \widetilde{N} / X^{t+1} \widetilde{N}$ and hence is finitely generated by $1(\mathrm{~b})$. Therefore $\widetilde{M}$ is finitely generated.

Recall that the graded Jacobson radical $J^{g}(\widetilde{T})$ of a graded $\widetilde{R}$-module $\widetilde{T}$ (note that we do not assume that $\widetilde{T}$ comes from some $T \in R$-filt but we stick to this notation with $\sim$ for $\widetilde{R}$-modules, hoping that it will be clear from the context whether the module is in $\mathscr{F}_{X}$ or not, i.e. when it comes from a module in $R$-filt or not) is defined to be the intersection of the gr-maximal submodules of $\widetilde{T}$, i.e. $J^{g}(\widetilde{R})$ is the largest proper graded ideal of $\widetilde{R}$ such that $1+a r$ is invertible for all $a \in J^{g}(\widetilde{R}) \cap \widetilde{R}_{0}$ and $r \in \widetilde{R}_{0}$. Basic properties of $J^{g}$ may be looked up in [6, Lemma I.7.4, p. 53].

2.7. Proposition. The following statements are equivalent for a filtered ring $R$ :

(1) $F_{-1} R \subset J\left(F_{0} R\right)$.

(2) $X \in J^{g}(\widetilde{R})$.

(3) For a finitely generated $\widetilde{M} \in \widetilde{R}-\mathrm{gr}, X \widetilde{M}=\widetilde{M}$ if and only if $\widetilde{M}=0$.

(4) For a finitely generated $\widetilde{M} \in \mathscr{F}_{X}, X \widetilde{M}=\widetilde{M}$ if and only if $\widetilde{M}=0$.

(5) If $M \in R$-filt has good filtration then $G(M)=0$ if and only if $M=0$.

(6) $F R$ is a faithful filtration.

If $\widetilde{R}$ is left Noetherian then the foregoing conditions are all equivalent to the properties mentioned in Theorem 1.8 .

Proof. (1) $\Leftrightarrow(2)$ Clearly $F_{-1} R \subset J\left(F_{0} R\right)$ if and only if $1+\left(F_{-1} R\right) X \subset U\left(\widetilde{R}_{0}\right)$ if and only if $X \in J^{g}(\widetilde{R})$.

(2) $\Leftrightarrow(3)$ The graded version of Nakayama's lemma, cf. Lemma I.7.5, p. 54 in $[6]$.

(3) $\Rightarrow(4)$ Obvious.

(4) $\Rightarrow$ (3) Let $\widetilde{M}$ be any finitely generated graded $\widetilde{R}$-module such that $X \widetilde{M}=\widetilde{M}$ and let $t_{X^{\infty}}(\widetilde{M})$ be the submodule $\left\{m \in \widetilde{M}, X^{n} m=0\right.$ for some $n \in \mathbf{N}\}$.

From the exact diagram in $\widetilde{R}$-gr:

$$
\begin{array}{ccc}
0 \rightarrow t_{X^{\infty}}(\widetilde{M}) & \rightarrow \widetilde{M} & \rightarrow \widetilde{M} / t_{X^{\infty}}(\widetilde{M}) \rightarrow 0 \\
\downarrow & \downarrow & \downarrow \\
0 & \rightarrow t_{X^{\infty}}(\widetilde{M}) \rightarrow \widetilde{M} \rightarrow \widetilde{M} / t_{X^{\infty}}(\widetilde{X}) \rightarrow 0
\end{array}
$$

where the vertical arrows are given by multiplication by $X$, we derive that $\widetilde{M} / t_{X^{\infty}}(\widetilde{M})=0$ using hypothesis (4). Hence $\widetilde{M}$ is $X$-torsion but as $\widetilde{M}$ is finitely generated this means that $X^{n} \widetilde{M}=0$ for a suitable $n \in \mathbf{N}$; however $\widetilde{M}=X \widetilde{M}=\cdots=X^{n} \widetilde{M}$, thus $\widetilde{M}=0$. 
(4) $\Leftrightarrow$ (5) Just the translation for the category equivalence $R$-filt $\leftrightarrow \mathscr{F}_{X}$.

(5) $\Leftrightarrow(6)$ Definition of faithful filtration.

After these results we may conclude that the Zariskian condition, equivalent to $\widetilde{R}$ being Noetherian and $F_{-1} R \subset J\left(F_{0} R\right)$, is the appropriate generalization of the notion of Zariski ring in commutative algebra, i.e. a Noetherian commutative ring $C$ with an $I$-adic filtration for an ideal $I$ of $C$ contained in the Jacobson radical $J(C)$. However, as far as microlocalization is concerned the weaker condition that $\widetilde{R}$ is (left) Noetherian will suffice to guarantee the good behavior of the microlocalization functor. Before dealing with these problems, let us look at the compatibility between the functor $\sim$ and the completion functor $\wedge$.

Consider $\widetilde{M} \in \mathscr{F}_{X}$ and let $(\widetilde{M})^{\wedge g}$ be the graded completion of $\widetilde{M}$ at the graded ideal $I$ of $\widetilde{R}$,

$$
(\widetilde{M})^{\wedge g}=\underbrace{\lim ^{g}}_{n} \widetilde{M} / X^{n} \widetilde{M}
$$

the inverse limit taken in $\widetilde{R}$-gr. Now calculate

$$
(\widetilde{M})_{p}^{\wedge g}={\underset{\varliminf}{n}}_{\lim _{p}} F_{p} M / F_{p-n} M={\underset{r \leq p}{\lim }}_{r \leq p} F_{p} M / F_{r} M
$$

but the latter is exactly $F_{p} \widehat{M}$ for the filtration $F \widehat{M}$ defined on the completion of $M$ at $F M$ (this is well known, see the bottom line of p. 283 of [6]), hence we have established

\subsection{Proposition. Let $M$ be a filtered R-module, then}

$$
(\widehat{M})^{\sim}=(\widetilde{M})^{\wedge g}={\underset{\lim }{n}}^{g} \widetilde{M} / X^{n} \widetilde{M} .
$$

In the terminology we are about to introduce in $\S 3$, the foregoing proposition just states that microlocalization at the trivial multiplicative set $\{1\}$ is nothing but the completion.

\section{Algebraic microlocalization}

Let $R$ be a separated filtered ring. Consider a multiplicatively closed subset $S$ (containing 1) in $R$. If $x \in F_{n} R-F_{n-1} R$ we let $\sigma(x)$ be the image of $x$ in $G(R)_{n}$. On $S$ we put this condition that $\sigma(S)=\{\sigma(s), s \in S\}$ is multiplicatively closed too. It then follows that $\sigma(s t)=\sigma(s) \sigma(t)$ for all $s, t \in S$. Clearly $\sigma(S)$ consists of homogeneous elements in $G(R)$. We construct $\widetilde{S}$ in $\widetilde{R}$ as follows: if $s \in S$ is such that $\sigma(s) \in G(R)_{n}$ then we let $\tilde{s} \in \widetilde{R}_{n}$ be the element $s \in F_{n} R$ but viewed as an element in $\bigoplus_{n \in \mathrm{Z}} F_{n} R$.

3.1. Lemma. The set $\widetilde{S}$ is multiplicatively closed in $\widetilde{R}$.

Proof. Since $\sigma(S)$ is multiplicatively closed it follows that $\sigma(s t)=\sigma(s) \sigma(t)$ for every $s, t \in S$ and therefore $\tilde{s} t=\tilde{s} \tilde{t}$ in $\widetilde{R}$ follows. Hence $\tilde{s} \tilde{t} \in \widetilde{S}$ and the claim is proved. 
3.2. Lemma. If $\sigma(S)$ satisfies the left Ore conditions in $G(R)$ then for each $n \in \mathbf{N}, \widetilde{S}$ maps to a homogeneous left Ore set $\bar{S}$ in $\widetilde{R} / I^{n}$.

Proof. Suppose first that $\bar{r} \bar{S}=0$ in $\widetilde{R} / I^{n}$, where $\bar{S}$ and $\bar{r}$ are the images of $\tilde{s} \in \widetilde{S}$ and $\tilde{r} \in \widetilde{R}$, resp. The Ore conditions for $\sigma(S)$ entail that there is an $\tilde{s}_{1} \in \widetilde{S}$ such that $\bar{s}_{1} \bar{r}=\bar{X} \bar{u}_{1}$, some $\bar{u}_{1} \in \widetilde{R} / I^{n}$. From $\bar{s}_{1} \bar{r} \bar{s}=0$ we obtain that $X u_{1} s=X^{n} b$ in $\bar{R}$, hence $\bar{u}_{1} \bar{s}=0$ in $\widetilde{R} / I$ and using the Ore conditions again we obtain an $\bar{s}_{2}$ with $\tilde{s}_{2} \in \widetilde{S}$ such that $\bar{s}_{2} \bar{u}_{1}=\bar{X} \bar{u}_{2}$ with $\bar{u}_{2} \in \widetilde{R} / I^{n}$. Consequently, $\bar{s}_{2} \bar{s}_{1} \bar{r}=\bar{X}^{2} \bar{u}_{2}$ and repeating this procedure, taking into account that $\bar{X}^{n}=0$ in $\widetilde{R} / I^{n}$, we arrive at $\bar{s}_{n} \cdots \bar{s}_{1} \bar{r}=0$ with $\tilde{s}_{n} \cdots \tilde{s}_{1} \in \widetilde{S}$. So we verified the first Ore condition for $\bar{S}$ in $\widetilde{R} / I^{n}$. For the second Ore condition we consider $\bar{s}, \bar{r} \in \widetilde{R} / I^{n}$ for $\bar{s}$ an image of some $\tilde{s} \in \widetilde{S}$ and $\bar{r}$ arbitrary. We have to establish the existence of $\bar{s}_{1}, \bar{r}_{1}$ such that $\bar{s}_{1} \bar{r}=\bar{r}_{1} \bar{s}$, with $\bar{s}_{1}$ an image of an $\tilde{s}_{1}$ in $\widetilde{S}$. Using the second Ore condition in $\widetilde{R} / I$ it is clear that we may find $\overline{s^{\prime}}, \bar{t}$ in $\widetilde{R} / I^{n}$ with $\bar{s}^{\prime}$ in $\bar{S}$ such that $\overline{s^{\prime}} \bar{r}=\bar{t} \bar{s}+\bar{X} \bar{u}_{1}, \bar{u}_{1} \in \widetilde{R} / I^{n}$. Repetition of the same argument leads to $\bar{s}^{\prime \prime} \bar{u}_{1}=\bar{a}_{1} \bar{s}+\bar{X} \bar{u}_{2}$ with $\bar{a}_{s}, \bar{u}_{2} \in \widetilde{R} / I^{n}$. Hence

$$
\overline{s^{\prime \prime} s^{\prime}} \bar{r}=\overline{s^{\prime \prime}} \bar{t} \bar{s}+\bar{X} \bar{a}_{1} \bar{s}+\bar{X}^{2} \bar{u}_{2}=\left(\overline{s^{\prime \prime}} \bar{t}+\bar{X} \bar{a}_{1}\right) \bar{s}+\bar{X}^{2} \bar{u}_{2} .
$$

After at most $n-1$ repetitions we arrive at $\bar{s}_{1} \bar{r}=\bar{r}_{1} \bar{s}$ with $\bar{s}_{1}$ equal to the product of $\overline{s^{\prime}}, \overline{s^{\prime \prime}}, \ldots$ (in the correct order) and $\bar{r}_{1}$ the element in $\widetilde{R} / I^{n}$ obtained at the final step.

For every $n \in \mathbf{N}$ we may define $Q \frac{g}{S}\left(\widetilde{R} / I^{n}\right)$ obtained by inverting the homogeneous left Ore set $\bar{S}$ of $\widetilde{R} / I^{n}$ in the classical way. We obtain an inverse system of graded morphisms in $\widetilde{R}$-gr: $\left\{Q_{\bar{S}}^{g}\left(\widetilde{R} / I^{n}\right) \rightarrow Q \frac{g}{S}\left(\widetilde{R} / I^{n-1}\right), n \in \mathbf{N}\right\}$, so we can take its inverse limit in $\widetilde{R}$-gr and we write

$$
\widetilde{Q}_{\widetilde{S}}^{\mu}(\widetilde{R})={\underset{\lim }{n}}^{g} Q \frac{g}{S}\left(\widetilde{R} / I^{n}\right)
$$

(caution: $\bar{S}$ depends on $n$ ) for the graded ring (and $\widetilde{R}$-module) we obtain this way. For a filtered $R$-module $M$ we define

$$
\widetilde{Q}_{\widetilde{S}}^{\mu}(\widetilde{M})={\underset{n}{n}}_{\lim ^{g}} Q_{\bar{S}}^{g}\left(\widetilde{M} / I^{n} \widetilde{M}\right)
$$

and it is not hard to verify that this defines a graded $\widetilde{Q}_{\widetilde{S}}^{\mu}(\widetilde{R})$-module.

This construction may be generalized considerably. To a kernel functor $\kappa$ on $R$-mod (cf. [6] for some details on localization theory), we correspond $\tilde{\kappa}$ on $\widetilde{R}$-mod by defining the Gabriel filter $\mathscr{L}(\tilde{\mathcal{K}})$ to be generated by the graded left ideals $L$ of $\widetilde{R}$ such that $L=\widetilde{H}$ for some $H \in \mathscr{L}(\kappa)$ (it is not hard to verify that the family of graded left ideals in $\mathscr{L}(\tilde{\kappa})$ is a basis for the filter and that we could have defined $\tilde{\kappa}$ to be the kernel functor on $\widetilde{R}$-mod corresponding to a "rigid graded kernel functor" $\tilde{\kappa}$ on $\widetilde{R}$-gr as defined in [6]). For every $n \in \mathbf{N}$ we define $\kappa^{(n)}$ to be the (graded) kernel functor on $\widetilde{R} / I^{n}$ induced by the graded 
ring morphism $j^{(n)}: \widetilde{R} \rightarrow \widetilde{R} / I^{n}$ (i.e. an $\widetilde{R} / I^{n}$-module $T$ will be $\kappa^{(n)}$-torsion exactly then when $j_{*}^{(n)} T$ is $\tilde{\kappa}$-torsion). Let $Q_{n}^{g}$ be the localization functor on $\widetilde{R} / I^{n}$-gr corresponding to $\kappa^{(n)}$, then define

$$
\widetilde{Q}_{\tilde{\kappa}}^{\mu}(\widetilde{M})=\underbrace{\lim ^{g}}_{n} Q_{n}^{g}\left(\widetilde{M} / I^{n} \widetilde{M}\right)
$$

and so we arrive at the microlocalization at $\kappa, Q_{\kappa}^{\mu}(M)$, in a way similar to what will follow for $Q_{S}^{\mu}$. We do not dwell upon this material here but we point out that iı has some use in particular cases where $\kappa$ corresponds to an open set in the Zariski topology on some projective variety or when $\kappa$ corresponds to the generically closed set of height one prime ideals of a ring over which differential operators are being considered.

3.3. Lemma. With notations as above $\widetilde{Q}_{\widetilde{S}}^{\mu}(\widetilde{M})$ is in $\mathscr{F}_{X}$.

Proof. If $a \in \widetilde{Q}_{\widetilde{S}}^{\mu}(\widetilde{M})$ is annihilated by $X$ then $X a_{(n)}=0$ for $0 \neq a_{(n)} \in$ $Q \frac{g}{S}\left(\widetilde{M} / I^{n} \widetilde{M}\right)$ representing $a$. There is an $\bar{s}$ in the image of $\widetilde{S}$ in $\widetilde{R} / I^{n}$ such that $X \bar{s} a_{(n)}=0$ with $\bar{s} a_{(n)} \neq 0$ in $\operatorname{Im} \varphi, \varphi: \widetilde{M} / I^{n} \widetilde{M} \rightarrow Q \frac{g}{s}\left(\widetilde{M} / I^{n} \widetilde{M}\right)$ the natural localization homomorphism. If $b_{(n)} \in \widetilde{M} / I^{n} \widetilde{M}$ represents $\bar{s} a_{(n)}$ then $b_{(n)}$ is not $\bar{S}$-torsion since $\bar{s} a_{(n)}$ is not $\bar{S}$-torsion. From $X \bar{s} a_{(n)}=0$ it then follows that $\bar{s}_{1} X b_{(n)}=0$ hence $\bar{s}_{1} b_{(n)} \in I^{n-1} \widetilde{M} / I^{n} \widetilde{M}$ and $b_{(n)}$ as well as $a_{(n)}$ must then be in $Q \frac{g}{S}\left(I^{n-1} \widetilde{M} / I^{n} \widetilde{M}\right)$, i.e. $a_{(n-1)}=0$. Since this argument works for any $n$ it follows that $a=0$ and therefore $\widetilde{Q}_{\widetilde{S}}^{\mu}(\widetilde{M})$ is $X$-torsionfree.

3.4. Corollary. To the X-torsionfree graded $\widetilde{R}$-module $\widetilde{Q}_{\widetilde{S}}^{\mu}(\widetilde{M})$ there corresponds a filtered $R$-module $Q_{S}^{\mu}(M)$ obtained as

$$
Q_{S}^{\mu}(M)=\widetilde{Q}_{\widetilde{S}}^{\mu}(\widetilde{M}) /(X-1) \widetilde{Q}_{\widetilde{S}}^{\mu}(\widetilde{M}) .
$$

The ring $Q_{S}^{\mu}(R)$, thus obtained, is called the microlocalization of $R$ at $S$. Similarly, $Q_{S}^{\mu}(M)$ is the microlocalization of $M$ and it is a filtered $R$-module. We have to justify the terminology introduced above by verifying that $Q_{S}^{\mu}(R)$ satisfies the universal property characterizing the microlocalization in the sense of $[8]$ a.o.

3.5. Proposition. Let $R$ be a separated filtered ring, let $j_{S}$ be the canonical ring morphism $R \rightarrow Q_{S}^{\mu}(R)$. Given a complete filtered ring $B$ and a filtered ring homomorphism $h: R \rightarrow B$ such that for all $s \in S, h(s)$ is invertible in $B$ with $h(s)^{-1} \in F_{-n} B$ if $\sigma(s) \in G(R)_{n}$. There exists a unique filtered ring homomorphism $g: Q_{S}^{\mu}(R) \rightarrow B$ such that $h$ factorizes as $R \rightarrow{ }_{j_{S}} Q_{S}^{\mu}(R) \rightarrow{ }_{g} B$. Proof. Since $h$ is a filtered morphism it yields a map of graded $\widetilde{R}$-modules $\tilde{h}: \widetilde{R} \rightarrow \widetilde{B}$, which is of degree zero. From $h\left(1_{R}\right)=1_{B}$ it follows that $\tilde{h}(X)=$ $X_{B}$, where $X_{B}$ is $1_{B}$ viewed as an element of $\widetilde{B}{ }_{1}$. It is trivial to verify that $\widetilde{B}$ 
is in fact a ring and that $\tilde{h}$ is a graded ring morphism. From $\tilde{h}\left(I^{n}\right)=X_{B}^{n} \tilde{h}(\widetilde{R})$ it follows that $\bar{h}$ induces graded morphisms $\widetilde{R} / I^{n} \rightarrow \tilde{h}(\bar{R}) / X_{B}^{n} \tilde{h}(\widetilde{R}) \rightarrow \widetilde{B} / I_{B}^{n}$, where $I_{B}=\widetilde{B} X_{B}$.

Now note that an $s \in S$ with $\sigma(s) \in G(R)_{n}$ has $h(s)^{-1}$ in $F_{-n} B-F_{-n-1} B$ because $h(s)^{-1} \in F_{-n-1} B$ leads to $1=h(s) h(s)^{-1} \in F_{n} B F_{-n-1} B \subset F_{-1} B$, a contradiction, while $h(s)^{-1} \in F_{-n} B$ holds by assumption. The assumptions on $S$ entail that $\widetilde{S}$ maps to left Ore sets $\bar{S} \subset \widetilde{R} / I^{n}, \overline{S_{h}}=\overline{\tilde{h}(\widetilde{S})} \subset \tilde{h}(\widetilde{R}) / X_{B}^{n} \tilde{h}(\widetilde{R})$ and the image of $h(\widetilde{S})$ in $\widetilde{B} / I_{B}^{n}$ is invertible in $\widetilde{B} / I_{B}^{n}$ because $\widetilde{h(S)}$ is invertible in $\widetilde{B}$. Note that the latter fact really depends on the observation that $h(s)^{-1} \notin$ $F_{-n-1} B$ when $\sigma(s) \in G(R)_{n}$ ! After localization at the left Ore sets involved we arrive at graded ring homomorphisms:

$$
Q \frac{g}{S}\left(\widetilde{R} / I^{n}\right) \rightarrow Q_{\bar{S}_{h}}^{g}\left(\tilde{h}(\widetilde{R}) / X_{B}^{n} \tilde{h}(\widetilde{R})\right) \rightarrow \widetilde{B} / I_{B}^{n} .
$$

Taking inverse limits in the graded sense leads to graded morphisms:

$$
\tilde{g}: \widetilde{Q}_{\widetilde{S}}^{\mu}(\widetilde{R}) \rightarrow \widetilde{Q}_{\widetilde{S}_{h}}^{\mu}(\tilde{h}(\widetilde{R})) \rightarrow \widetilde{B}=\underbrace{\lim ^{g}}_{n} \widetilde{B} / I^{n} B .
$$

The filtered morphism $g: Q_{S}^{\mu}(R) \rightarrow B$ corresponding to $\tilde{g}$ is also a ring morphism (not only an $R$-module morphism) and from $\widetilde{R} \rightarrow_{j_{s}} \widetilde{Q}_{\widetilde{S}}^{\mu}(\widetilde{R}) \rightarrow \widetilde{B}$ we obtain $R \rightarrow{ }_{j_{s}} Q_{S}^{\mu}(R) \rightarrow_{g} B$ factorizing $h$ as desired (this is trivial to check).

3.6. Corollary. (1) $Q_{S}^{\mu}(R)$ is the microlocalization of $R$ at $S$ in the sense of [8].

(2) A similar proof establishes the same result for $Q_{S}^{\mu}(M)$.

(3) The fact that we keep track of the intermediate ring $\widetilde{Q}_{\widetilde{S}_{h}}^{\mu}(\tilde{h}(\widetilde{R}))$ in the proof shows that $Q_{S_{h}}^{\mu}(h(R))$ is a ring and that $Q_{S}^{\mu}(R) \rightarrow Q_{S_{h}}^{\mu}(h(R))$ is a filtered ring homomorphism.

(4) The equality of norms as in Lemma 5.16 and Corollary 5.20 in [8] is a consequence of our proof above, in fact here it follows from the very simple fact that $h(s)^{-1} \in F_{-n} B$ entails $h(s)^{-1} \notin F_{-n-1} B$. This marks the advantage of our method over the cumbersome norm-calculations used in [8].

3.7. Lemma. $F Q_{S}^{\mu}(M)$ is separated.

Proof. Application of Lemma 2.2(1) shows that we only have to check whether $\cap_{m} X^{m} \widetilde{Q}_{\widetilde{S}}^{\mu}(\widetilde{M})=0$. But

$$
\begin{aligned}
& \bigcap_{m} X^{m} \widetilde{Q}_{\widetilde{S}}^{\mu}(\widetilde{M})=\underbrace{\lim ^{g}}_{m} X^{m} \underbrace{\lim ^{g}}_{n} \widetilde{Q} \frac{g}{S}\left(\widetilde{M} / X^{n} \widetilde{M}\right) \\
& =\underbrace{\lim _{m}^{g} \underbrace{\lim ^{g}}_{n} X^{m} \widetilde{Q}}_{m} \widetilde{S}\left(\widetilde{M} / X^{n} \widetilde{M}\right) \\
& =\underbrace{\lim ^{g}}_{n} \underbrace{\lim ^{g}}_{m} X^{m} \widetilde{Q} \frac{g}{S}\left(\widetilde{M} / X^{n} \widetilde{M}\right)=0 .
\end{aligned}
$$


At this point we have to introduce a condition that enables us to calculate $\mathrm{lim}$ in an easier way, i.e. making it right exact in some easy recognizable cases. If one is given a sequence of homomorphisms of inverse systems $0 \rightarrow\left(A_{n}\right) \rightarrow$ $\left(B_{n}\right) \rightarrow\left(C_{n}\right) \rightarrow 0$ then we say that the system is exact if we have exact sequences $0 \rightarrow A_{n} \rightarrow B_{n} \rightarrow C_{n} \rightarrow 0$ for each $n$. For an exact sequence $0 \rightarrow\left(A_{n}\right) \rightarrow$ $\left(B_{n}\right) \rightarrow\left(C_{n}\right) \rightarrow 0$ we have that $0 \rightarrow \lim _{n} A_{n} \rightarrow \lim _{n} B_{n} \rightarrow \lim _{n} C_{n}$ is also exact. If $\left(A_{n}\right)$ satisfies the Mittag-Leffer condition (M.L.) then it also follows that

$$
0 \rightarrow \underset{n}{\lim } A_{n} \rightarrow \underset{n}{\lim } B_{n} \rightarrow \underset{n}{\lim } C_{n} \rightarrow 0
$$

is exact.

Recall M.L. (cf. p. 191 in [5]): the inverse system $\left(A_{n}\right)$ satisfies M.L. if for each $n$, the decreasing family of subgroups of $A_{n}\left\{\varphi_{n^{\prime} n}\left(A_{n^{\prime}}\right) \subset A_{n}, n^{\prime} \geq n\right\}$ is stationary; in other words for each $n$ there exists an $n_{0} \geq n$ such that for all $n^{\prime}, n^{\prime \prime} \geq n_{0}, \varphi_{n^{\prime} n}\left(A_{n^{\prime}}\right)=\varphi_{n^{\prime \prime} n}\left(A_{n^{\prime \prime}}\right)$, where the $\varphi_{n^{\prime} n}$ are morphisms in the inverse system.

3.8. Lemma (cf. [5, Proposition 9.1 and Example 9.1.1]). Let there be given a short exact sequence of inverse systems of abelian groups:

$$
0 \rightarrow\left(A_{n}\right) \rightarrow\left(B_{n}\right) \rightarrow\left(C_{n}\right) \rightarrow 0 .
$$

(1) If $\left(B_{n}\right)$ satisfies M.L. so does $\left(C_{n}\right)$.

(2) If $\left(A_{n}\right)$ satisfies M.L. then $0 \rightarrow \lim A_{n} \rightarrow \underset{\lim }{ } B_{n} \rightarrow \underset{\lim }{\longleftarrow} C_{n} \rightarrow 0$ is an exact sequence.

(3) If all the maps $\varphi_{n^{\prime} n}: A_{n^{\prime}} \rightarrow A_{n}$ are surjective then $\left(A_{n}\right)$ satisfies M.L.

When considering inverse systems of graded objects one may consider a weaker version of the M.L. condition, i.e. the 'graded M.L. condition' by restricting attention to the system $\varphi_{n^{\prime} n}^{t} \mid\left(A_{n^{\prime}}\right)_{t}$ for a fixed degree $t \in \mathbf{Z}$. However in the sequel we will use only the full M.L. condition.

3.9. Proposition. $F Q_{S}^{\mu}(M)$ is complete, for $M \in R$-filt .

Proof. It is enough to show that $\widetilde{Q}_{\widetilde{S}}^{\mu}(\widetilde{M})$ is graded $(X)$-adically complete. I.e.

$$
\widetilde{Q}_{\widetilde{S}}^{\mu}(\widetilde{M})={\underset{m}{\lim }}^{g} \widetilde{Q}_{\widetilde{S}}^{\mu}(\widetilde{M}) / X^{m} \widetilde{Q}_{\widetilde{S}}^{\mu}(\widetilde{M}) \text {. }
$$

But

$$
\begin{aligned}
& \underbrace{\lim _{m}^{g}}_{m} \widetilde{Q}_{\widetilde{S}}^{\mu}(\widetilde{M}) / X^{m} \widetilde{Q}_{\widetilde{S}}^{\mu}(\widetilde{M}) \\
& =\underbrace{\lim _{m}^{g}}_{m}(\underbrace{\lim ^{g}}_{n} \widetilde{Q} \frac{g}{S}\left(\widetilde{M} / X^{n} \widetilde{M}\right)) /(\underbrace{\lim ^{g}}_{n} X^{m} \widetilde{Q}_{\bar{S}}^{g}\left(\widetilde{M} / X^{n} \widetilde{M}\right)) \\
& =\underbrace{\lim _{m}^{g}}_{m} \underbrace{\lim ^{g}}_{n} \widetilde{Q} \frac{g}{S}\left(\widetilde{M} / X^{n} \widetilde{M}\right) / X^{m} \widetilde{Q} \frac{g}{S}\left(\widetilde{M} / X^{n} \widetilde{M}\right)(M L !) \\
& =\underbrace{\lim _{n}^{g}}_{n}{\underset{m}{\lim }}^{g} \widetilde{Q} \frac{g}{S}\left(\widetilde{M} / X^{n} \widetilde{M}\right) / X^{m} \widetilde{Q} \frac{g}{S}\left(\widetilde{M} / X^{n} \widetilde{M}\right)=\widetilde{Q}_{\widetilde{S}}^{\mu}(\widetilde{M}) \text {. }
\end{aligned}
$$


3.10. Proposition. The associated graded module of $Q_{S}^{\mu}(M)$ is $G\left(Q_{S}^{\mu}(M)\right)=$ $\sigma(S)^{-1} G(M)$.

Proof. To find $G\left(Q_{S}^{\mu}(M)\right)$ we have to compute $\widetilde{Q}_{\widetilde{S}}^{\mu}(\widetilde{M}) / X \widetilde{Q}_{\widetilde{S}}^{\mu}(\widetilde{M})$. But

$$
\begin{aligned}
& \widetilde{Q}_{\widetilde{S}}^{\mu}(\widetilde{M}) / X \widetilde{Q}_{\widetilde{S}}^{\mu}(\widetilde{M})=\underbrace{\lim _{n}^{g}}_{n} \widetilde{Q}_{\bar{S}}^{g}\left(\widetilde{M} / X^{n} \widetilde{M}\right) / \overleftarrow{\lim }^{g} X \widetilde{Q}_{\bar{S}}^{g}\left(\widetilde{M} / X^{n} \widetilde{M}\right) \\
& =\underbrace{\lim _{n}^{q}}_{n} \widetilde{Q}_{\bar{S}}^{g}\left(\widetilde{M} / X^{n} \widetilde{M}\right) / X \widetilde{Q}_{\bar{S}}^{g}\left(\widetilde{M} / X^{n} \widetilde{M}\right)(M L !) \\
& =\widetilde{Q}_{\bar{S}}^{g}(\widetilde{M} / X \widetilde{M})=\sigma(S)^{-1} G(M) . \quad \square
\end{aligned}
$$

3.11. Remarks. 1. It is clear that $Q_{S}^{\mu}$ is a functor from $R$-filt $\rightarrow Q_{S}^{\mu}(R)$-filt . Indeed, it is clear that $Q_{\bar{S}}^{g}\left(\widetilde{M} / I^{n} \widetilde{M}\right)$ is a graded $Q \frac{g}{S}\left(\widetilde{R} / I^{n}\right)$-module for each $n$, so it is also clear that $Q_{S}^{\mu}(M)$ is a $Q_{S}^{\mu}(R)$-module. Similarly one verifies the action of $Q_{S}^{\mu}$ on filtered morphisms.

2. If $S$ is an Ore set of $R$ and $F R$ is trivial then

$$
\widetilde{R}=R[X], \quad G(R)=R, \quad \widetilde{Q}_{\widetilde{S}}^{\mu}(\widetilde{R})={\underset{n}{\lim }}^{g} S^{-1}\left(R[X] / X^{n}\right) \stackrel{(!)}{=}\left(S^{-1} R\right)[X]
$$

and

$$
Q_{S}^{\mu}(R)=\left(S^{-1} R\right)[X] /(X-1)=S^{-1} R .
$$

3. If $S$ is an Ore set of $R$ and $F R$ is any separated filtration then

$$
\widetilde{Q}_{\widetilde{S}}^{\mu}(\widetilde{R})={\underset{n}{\lim }}^{g} \bar{S}^{-1}\left(\widetilde{R} / I^{n}\right)={\underset{\lim }{n}}^{g} \bar{S}^{-1} \widetilde{R} / \bar{S}^{-1} I^{n}=\left(S^{-1} \widetilde{R}\right)^{\wedge}
$$

where $\wedge$ is the graded $S^{-1} I$-adic completion. We obtain that $Q_{S}^{\mu}(R)=$ $\left(S^{-1} R\right)^{\wedge\left(F_{S}\right)}$ where $\wedge\left(F_{S}\right)$ is a completion corresponding to the $S$-closure of the filtration $F$. This $F_{S}$ corresponds to the pseudo-norm associated to $S$ in [8].

4. If $S=\{1\}$ then $Q_{1}^{\mu}(R)=\widehat{R}$ follows from 3 .

So far we have obtained short elegant proofs of most important properties of $Q_{S}^{\mu}$. Now we continue with a more systematic study of the funtor $Q_{S}^{\mu}$.

For a graded $\widetilde{R}$-module $T, T(n)$ with $n \in \mathbf{Z}$ stands for the shifted graded module, i.e. the $\widetilde{R}$-module $T$ with gradation shifted over $n$. We introduce the notation $t_{X^{n}}(T)=\left\{t \in T, X^{n} t=0\right\}$ and $t_{X^{\infty}}(T)=\left\{t \in T, X^{n} t=0\right.$ for some $n \in \mathbf{Z}\}$. We write $\left(t_{X^{n}}(T)(-n)\right)_{n}$ for the inverse system defined by the graded morphisms $\varphi_{n^{\prime}, n}: t_{X^{n}}(T)(-n) \rightarrow t_{X^{n^{\prime}}}(T)\left(-n^{\prime}\right)$, being multiplication by $X^{n^{\prime}-n}$.

We view $t_{X^{n}}(T)$ as an $\widetilde{R} / I^{n}$-module in the obvious way. Then it makes sense to consider the inverse system (again graded maps):

$$
\varphi_{n^{\prime}, n}: Q \frac{g}{S}\left(t_{X^{n}}(T)(-n)\right) \rightarrow Q \frac{g}{S}\left(t_{X^{n^{\prime}}}(T)\left(-n^{\prime}\right)\right) .
$$

An $\widetilde{R}$-module $\widetilde{M}$ is said to be prefinite if there is an $m \in \mathbf{N}$ such that $X^{m} t_{X^{\infty}}(\widetilde{M})=0$. 
3.12. Lemma. If $\widetilde{M}$ is prefinite then the inverse system

$$
\left(Q_{\bar{S}}^{g}\left(t_{X^{n}}(\widetilde{M})(-n)\right)\right)_{n}
$$

satisfies M.L. and we have that its projective limit is zero.

Proof. Obvious.

3.13. Lemma. (1) Any finitely generated graded $X$-torsion module $T$ in $\widetilde{R}$-gr is prefinite.

(2) If $f: M \rightarrow N$ is a filtered morphism in $R$-filt such that there exists an $m \in \mathbf{N}$ such that $f\left(F_{n} M\right) \supset F_{n-m} N$ then $\operatorname{Coker}(\tilde{f})$ is prefinite.

Proof. (1) Trivial.

(2) $F_{p}(N / f(M))=F_{p} N / F_{p} f(M)=F_{p} N / f\left(F_{p} M\right)$, hence we have a surjective map $F_{p} N / F_{p-m} N \rightarrow F_{p}(N / f(M))$. Hence $\tilde{N} / X^{m} \tilde{N} \rightarrow$ Coker $\tilde{f}$ is surjective but then $\operatorname{Coker}(\tilde{f})$ is prefinite.

3.14. Corollary. If $F M$ and $F^{\prime} M$ are filtrations on $M$ such that the identity of $M$ satisfies Lemma 3.13(2), i.e. $F_{n} M \supset F_{n-m}^{\prime} M$ for all $n \in \mathbf{Z}$, then the cokernel of $\tilde{1}_{M}$ is prefinite.

Our first result concerning exactness properties of $Q_{S}^{\mu}$ can now be stated as follows.

3.15. Theorem. Let $0 \rightarrow \tilde{A} \rightarrow \widetilde{B} \rightarrow \widetilde{C} \rightarrow 0$ be an exact sequence of graded $\widetilde{R}$-modules such that either

(1) $\widetilde{C}$ is $X$-torsionfree (hence certainly prefinite),

(2) $\widetilde{B}$ and $\widetilde{C}$ are prefinite.

Then, with conventions on $S$ as before, the following sequence is exact (in $\widetilde{R}$-gr):

$$
0 \rightarrow \widetilde{Q}_{\widetilde{S}}^{\mu}(\widetilde{A}) \rightarrow \widetilde{Q}_{\widetilde{S}}^{\mu}(\widetilde{B}) \rightarrow \widetilde{Q}_{\widetilde{S}}^{\mu}(\widetilde{C}) \rightarrow 0 .
$$

Proof. There is an exact sequence (in $\widetilde{R}$-gr) of inverse systems:

$$
\begin{aligned}
0 & \rightarrow\left(Q \frac{g}{S}\left(t_{X^{n}}(\widetilde{A})(-n)\right)\right)_{n} \rightarrow\left(Q \frac{g}{S}\left(t_{X^{n}}(\widetilde{B})(-n)\right)\right)_{n} \\
& \rightarrow\left(Q \frac{g}{S}\left(t_{X^{n}}(\widetilde{C})(-n)\right)\right)_{n} \rightarrow\left(Q \frac{g}{S}\left(\widetilde{A} / I^{n} \widetilde{A}\right)\right)_{n} \\
& \rightarrow\left(Q \frac{g}{S}\left(\widetilde{B} / I^{n} \widetilde{B}\right)\right)_{n} \rightarrow\left(Q \frac{g}{S}\left(\widetilde{C} / I^{n} \widetilde{C}\right)\right)_{n} \rightarrow 0 .
\end{aligned}
$$

It now suffices to apply Lemmas 3.12 and 3.8, taking into account that in case (2) $A$ is also prefinite, and by taking $\lim$ we obtain the desired exact sequence.

3.16. Corollary. (1) If $0 \rightarrow A \rightarrow B \rightarrow C \rightarrow 0$ is a strict exact sequence of filtered $R$-modules then the sequence $0 \rightarrow Q_{S}^{\mu}(A) \rightarrow Q_{S}^{\mu}(B) \rightarrow Q_{S}^{\mu}(C) \rightarrow 0$ is also exact.

(2) If $F M$ and $F^{\prime} M$ are equivalent filtrations on $M$ then $F Q_{S}^{\mu}(M)$ and $F^{\prime} Q_{S}^{\mu}(M)$ are equivalent, i.e. the $R$-module $Q_{S}^{\mu}(M)$ does not depend on the chosen equivalent filtration on $M$. 
(3) If $\widetilde{R}$ is left Noetherian and $0 \rightarrow A \rightarrow B \rightarrow C \rightarrow 0$ is an exact sequence of filtered $R$-modules such that $\widetilde{A}, \widetilde{B}, \widetilde{C}$ are finitely generated $\widetilde{R}$-modules then the sequence

$$
0 \rightarrow Q_{S}^{\mu}(A) \rightarrow Q_{S}^{\mu}(B) \rightarrow Q_{S}^{\mu}(C) \rightarrow 0
$$

is an exact sequence of filtered $Q_{S}^{\mu}(R)$-modules.

Proof. (1) Follows from Theorem 3.15(1).

(2) We may apply Theorem 3.15(2) and Lemma 3.13(2), and use exactness of $-\otimes \widetilde{R} /(X-1) \widetilde{R}$ on $\widetilde{R}$-gr.

(3) Since $\widetilde{R}$ is left Noetherian, the complex $0 \rightarrow \widetilde{A} \rightarrow \widetilde{B} \rightarrow \widetilde{C} \rightarrow 0$ has finitely generated homology hence it is prefinite in view of Lemma 3.13(1) and clearly it is also $X$-torsion. By repeated application of Theorem 3.15(2) we obtain that the complex

$$
0 \rightarrow \widetilde{Q}_{\widetilde{S}}^{\mu}(\widetilde{A}) \rightarrow \widetilde{Q}_{\widetilde{S}}^{\mu}(\widetilde{B}) \rightarrow \widetilde{Q}_{\widetilde{S}}^{\mu}(\widetilde{C}) \rightarrow 0
$$

has $X$-torsion homology. Therefore

$$
0 \rightarrow Q_{S}^{\mu}(A) \rightarrow Q_{S}^{\mu}(B) \rightarrow Q_{S}^{\mu}(C) \rightarrow 0
$$

is an exact sequence of filtered $Q_{S}^{\mu}(R)$-modules.

We present the following result in a rather general form, its corollaries provide short and intrinsically graded proofs of a.o. Propositions 3.9 and 3.10.

3.17. Proposition. If $\widetilde{M}$ is a prefinite graded $\widetilde{R}$-module then

$$
\widetilde{R} / I^{n} \otimes_{\widetilde{R}} \widetilde{Q}_{\widetilde{S}}^{\mu}(\widetilde{M}) \cong Q_{\widetilde{S}}^{g}\left(\widetilde{M} / I^{n} \widetilde{M}\right) .
$$

Proof. Apply Theorem 3.15 to the exact sequence

$$
0 \rightarrow t_{X^{n}}(\widetilde{M}) \rightarrow \widetilde{M} \underset{X^{n}}{\rightarrow} \widetilde{M} \rightarrow \widetilde{M} / I^{n} \widetilde{M} \rightarrow 0
$$

3.18. Corollaries. (1) If $M \in R$-filt then $G\left(Q_{S}^{\mu}(M)\right)=\sigma(S)^{-1} G(M)$.

(2) If $S$ and $T$ are multiplicative sets in $R$ with properties as before and if $S V T$ is the multiplicative set generated by $S \cup T$ then let us assume $0 \notin$ $\sigma(S) V \sigma(T)$ (just to avoid triviality of the statements).

Then $Q_{S}^{\mu}\left(Q_{T}^{\mu}(M)\right) \cong Q_{S V T}^{\mu}(M)$. This follows from Proposition 3.17 and the fact that $Q_{\bar{S}}^{g} Q_{\bar{T}}^{g}=Q_{\overline{S V T}}^{g}$ on graded $\widetilde{R} / I^{n}$-modules.

3.19. Theorem. Let $\widetilde{R}$ be left Noetherian.

(1) If $\widetilde{M}$ is a graded finitely generated $\widetilde{R}$-module then

$$
\widetilde{Q}_{\widetilde{S}}^{\mu}(\widetilde{M})=\widetilde{Q}_{\widetilde{S}}^{\mu}(\widetilde{R}) \otimes_{\widetilde{R}} \widetilde{M} .
$$

(2) $\widetilde{Q}_{\widetilde{S}}^{\mu}(\widetilde{R})$ is flat.

Proof. (1) Since $\widetilde{Q}_{\widetilde{S}}^{\mu}$ commutes with finite direct sums the claim holds for gr-free graded $\widetilde{R}$-modules of finite rank. For $\widetilde{M}$ consider a presentation 
$\widetilde{G} \rightarrow \widetilde{F} \rightarrow \widetilde{M} \rightarrow 0$ where $\widetilde{F}, \widetilde{G}$ are gr-free of finite rank. Application of Theorem 3.15 yields

$$
\begin{gathered}
\widetilde{Q}_{\widetilde{S}}^{\mu}(\widetilde{G}) \rightarrow \widetilde{Q}_{\uparrow}^{\mu}(\widetilde{F}) \rightarrow \widetilde{Q}_{\widetilde{S}}^{\mu}(\widetilde{M}) \rightarrow 0 \\
\widetilde{Q}_{\widetilde{S}}^{\mu}(\widetilde{R}) \otimes_{\widetilde{R}} \widetilde{G} \rightarrow \widetilde{Q}_{\widetilde{S}}^{\mu}(\widetilde{R}) \otimes_{\widetilde{R}} \widetilde{F} \rightarrow \widetilde{Q}_{\widetilde{S}}^{\mu}(\widetilde{R}) \otimes_{\widetilde{R}} \widetilde{M} \rightarrow 0
\end{gathered}
$$

By the five lemma, the vertical map on the right is an isomorphism of graded $\widetilde{Q}_{\widetilde{S}}^{\mu}(\widetilde{R})$-modules.

(2) The functor $\widetilde{Q}_{\widetilde{S}}^{\mu}(\widetilde{R}) \otimes_{\widetilde{R}}$ - commutes with graded direct limits and it is exact on finitely generated modules by the first part and Theorem 3.15(2).

3.20. Corollary. Let $\widetilde{R}$ be left Noetherian, then:

(1) The functor $Q_{S}^{\mu}(R) \otimes_{R}$ - preserves strict maps and it is exact on $R$-modules.

(2) If $M \in R$-filt is filt-finitely generated, i.e. $\widetilde{M}$ is finitely generated, then $Q_{S}^{\mu}(R) \otimes_{R} M \cong Q_{S}^{\mu}(M)$ as filtered $R$-modules.

Proof. (1) Since $\widetilde{Q}_{\widetilde{S}}^{\mu}(\widetilde{R})$ is flat as an $\widetilde{R}$-module, flatness of $Q_{S}^{\mu}(R)$ follows for example from localization at $\left\{1, X, X^{2}, \ldots\right\}$. A strict morphism $f$ yields a graded morphism $\tilde{f}$ with $X$-torsionfree cokernel. Then the flatness of $\widetilde{Q}_{\widetilde{S}}^{\mu}(\widetilde{R})$ yields that $\widetilde{Q}_{\widetilde{S}}^{\mu}(\widetilde{R}) \otimes \tilde{f}$ has $X$-torsionfree cokernel too.

(2) Trivial from the foregoing.

3.21. Note. The second statement in the above corollary makes sense for nonfiltered modules. Indeed, if $M$ is a finitely generated $R$-module then we may define a good filtration on it and applying Corollary 3.20(2) to the filtered module thus defined yields an isomorphism of $R$-modules $Q_{S}^{\mu}(R) \otimes_{R} M \cong Q_{S}^{\mu}(M)$.

A multiplicative set $S$ is said to be saturated if $S=\{r \in R, \sigma(r) \in \sigma(S)\}$. J. E. Björk pointed out to us that M. Kashiwara made the following observation in his master's thesis: if $F R$ is Zariskian and $G(R)$ is a commutative Noetherian domain then any saturated $S$ is an Ore set. Now, making use of our approach via the ring $\widetilde{R}$ we can extend this result to the situation where $\widetilde{R}$ is Noetherian as follows. Put $\widetilde{S}_{\text {sat }}$ equal to the multiplicative set of homogeneous elements in $\widetilde{S}+\widetilde{R} X$; then it is clear that $\widetilde{S}_{\text {sat }}$ maps to $S_{\text {sat }}=\{r \in R, \sigma(r) \in \sigma(S)\}$ (for a multiplicatively closed set $S$ such that $\sigma(S)$ is multiplicatively closed) under the map $\widetilde{R} \rightarrow R=\widetilde{R} /(X-1) \widetilde{R}$. If $\widetilde{S}_{\text {sat }}$ is an Ore set in $\widetilde{R}$ then $S_{\text {sat }}$ is an Ore set in $R$.

3.22. Proposition. If $\widetilde{R}$ is left Noetherian and $S$ is a saturated multiplicatively closed set $R$ such that $\sigma(S)$ satisfies the second left Ore condition in $G(R)$ then $S$ satisfies the second left Ore condition in $R$. In case $\sigma(S)$ is a regular left Ore set in $G(R), S$ is a left Ore set in $R$ and $Q_{S}^{\mu}(R)=\left(S^{-1} R\right)^{\wedge_{F_{S}}}$.

Proof. Clearly $\widetilde{S}=\widetilde{S}_{\text {sat }}$ since $S$ is saturated. By Lemma 3.2, $\widetilde{S}$ maps to homogeneous sets satisfying the second left Ore condition in $\widetilde{R} / I^{n}$ for every $n \in \mathbf{N}$ (note: first or second refers to the order on the Ore conditions as used in Lemma 
3.2). Now $I$ is an invertible ideal of a Noetherian ring $\widetilde{R}$ and $I=\widetilde{R} X$ is centrally generated so it certainly satisfies the Artin-Rees condition with respect to left ideals $L$ of $\widetilde{R}, L \cap \widetilde{R} X^{h(n)} \subset L X^{n}$, for some $h(n)$ associated to $n \in \mathbf{N}$. Combining this Artin-Rees condition with the second left Ore conditions for $\bar{S}^{(n)}$, the image of $\widetilde{S}$ in $\widetilde{R} / \widetilde{R} X^{n}$, with $n \in \mathbf{N}$, one easily obtains the second left Ore condition for $\widetilde{S}$ in $\widetilde{R}$. It is obvious that the regularity of $\sigma(S)$ in $G(R)$ allows us to lift the first Ore condition as well. In view of Remark 3.11.3 it follows indeed that microlocalization at $S$ is just a completion of $S^{-1} R$ at the localized filtration.

3.23. Corollary. For any $S$ (with $\sigma(S)$ multiplicatively closed as always) we have $Q_{S}^{\mu}(R)=\left(S_{\mathrm{sat}}^{-1} R\right)^{\wedge} F_{S_{\mathrm{sat}}}$, i.e. microlocalization is always just a suitable completion of a localization at some Ore set in $R$.

\section{REFERENCES}

1. M. Awami and F. Van Oystaeyen, On filtered rings with Noetherian associated graded rings, Proc. Ring Theory Meeting (Granada, 1986), Springer-Verlag, Berlin and New York, 1987.

2. J. E. Björk, Rings of differential operators, Math. Library, vol. 21, North-Holland, Amsterdam, 1979.

3. __ Unpublished notes, 1985.

4. O. Gabber, On the integrability of the characteristic variety, Amer. J. Math. 103 (1981), 445468.

5. R. Hartshorne, Algebraic geometry, Springer-Verlag, Berlin, 1977.

6. C. Năstăsescu and F. Van Oystaeyen, Graded ring theory, Math. Library, vol. 28, NorthHolland, Amsterdam, 1980.

7. T. Springer, Micro-localization algébrique, Séminaire d'Algèbre Dubreil-Malliavin, Lecture Notes in Math., Springer-Verlag, Berlin, 1980.

8. A. Van den Essen, Algebraic micro-localization, Comm. Algebra 14 (1986), 971-1000.

9. Li Huishi and F. Van Oystaeyen, Zariskian filtrations, Comm. Algebra (to appear).

10. P. Shapira, Microdifferential systems in the complex domain, Grundlehren der Math. Wiss., no. 269, Springer-Verlag, Berlin, 1985.

Departamento de Algebra y Fundamentos, Universidad de Granada, Facultad de Ciencias Experimentales, Almeria, Spain (Current address of Maria J. Asensio)

Department of Mathematics and Computer Science, University of Antwerp, UiA, ANTwerp, Belgium (Current address of Michel Van den Bergh and Freddy Van Oystaeyen) 